\title{
Factors that affect patient reported outcome after anterior cruciate ligament reconstruction-a systematic review of the Scandinavian knee ligament registers
}

\author{
Eric Hamrin Senorski, ${ }^{1}$ Eleonor Svantesson, ${ }^{2}$ Angelo Baldari, ${ }^{3}$ Olufemi R Ayeni, ${ }^{4}$ \\ Lars Engebretsen, ${ }^{5,6}$ Francesco Franceschi, ${ }^{3}$ Jón Karlsson, ${ }^{2,7}{ }^{1}$ Kristian Samuelsson ${ }^{2,7}$
}

\begin{abstract}
- Additional material is published online only. To view please visit the journal online (http://dx.doi.org/10.1136/ bjsports-2017-098191).

${ }^{1}$ Department of Health and Rehabilitation, Institute of Neuroscience and Physiology, Sahlgrenska Academy, University of Gothenburg, Goteborg, Sweden ${ }^{2}$ Department of Orthopaedics, Institute of Clinical Sciences, Sahlgrenska Academy, University of Gothenburg, Goteborg, Sweden ${ }^{3}$ Department of Orthopaedic and Trauma Surgery, Campus Bio-Medico University of Rome, Rome, Italy

${ }^{4}$ Division of Orthopaedic Surgery, Department of Surgery, McMaster University, Hamilton, Ontario, Canada

${ }^{5}$ Oslo University Hospital and University of Oslo, Oslo, Norway ${ }^{6}$ Oslo Sports Trauma Research Centre, The Norwegian School of Sports Sciences, Oslo, Norway ${ }^{7}$ Department of Orthopaedics, Sahlgrenska University Hospital, Goteborg, Sweden
\end{abstract}

Correspondence to Dr. Eric Hamrin Senorski, Department of Orthopaedics, Institute of Clinical Sciences, Sahlgrenska Academy, University of Gothenburg, Gothenburg SE-431 80, Sweden;

eric.hamrin.senorski@gu.se

Received 19 June 2017 Published Online First 20 July 2018

Check for updates

(C) Author(s) (or their employer(s)) 2019. No commercial re-use. See rights and permissions. Published by BMJ.

To cite: Hamrin Senorski E, Svantesson E, Baldari A, et al. Br J Sports Med 2019:53:410-417.

\begin{abstract}
Objective To perform a systematic review of findings from the Scandinavian knee ligament registers with regard to factors that affect patient reported outcome after anterior cruciate ligament $(\mathrm{ACL})$ reconstruction.

Design Systematic review.

Data sources Four electronic databases: PubMed, EMBASE, the Cochrane Library and AMED were searched, and 157 studies were identified. Two reviewers independently screened the titles, abstracts and full text articles for eligibility. A modified version of the Downs and Black checklist was applied for quality appraisal. Eligibility criteria for selecting studies Studies published from the Scandinavian registers from their establishment in 2004 and onwards that documented patient reported outcome and provided information on concomitant injuries were eligible.

Results A total of 35 studies were included. Younger age at $A C L$ reconstruction, male sex, not smoking and receiving a hamstring tendon autograft positively influenced patient reported outcome. Patients with concomitant cartilage and meniscal injuries reported inferior subjective knee function compared with patients with an isolated $A C L$ tear. One study reported that patients treated non-reconstructively reported inferior knee function compared with patients who had ACL reconstruction.
\end{abstract}

Conclusion Younger age, male sex, not smoking, receiving a hamstring tendon autograft and the absence of concomitant injuries were associated with superior patient reported outcomes after $A C L$ reconstruction.

\section{INTRODUCTION}

Patient reported outcome is a cornerstone in understanding patients' perceptions of treatment outcome in medicine. As the Scandinavian knee ligament registers contain data from more than 70000 patients, they provide a unique opportunity to understand and interpret factors that affect patient reported outcome after anterior cruciate ligament (ACL) reconstruction. ${ }^{1}$ The Knee injury and Osteoarthritis Outcome Score ${ }^{2}$ (KOOS) is the common patient reported outcome used in the Sacndinavian knee ligament registers. The KOOS is collected preoperatively and at clinically relevant time points postoperatively. By collecting KOOS data, clinicians are able to monitor and compare the outcome for subgroups of patientsfor example, ACL reconstruction and rehabilitation compared with rehabilitation alone, or adolescents compared with adults. Understanding the factors that contribute to outcome in patients with ACL injury is of paramount importance for shared decision making between clinicians and the patient.

The purpose of this study was to (1) determine whether specific patient related and surgery related factors influenced patient reported outcome, (2) assess if there was a relationship between concomitant injuries (ie, meniscus tears and articular cartilage lesions) and patient reported outcomes, including to determine predictors of concomitant injuries, and (3) identify tools for interpretation of the results from patient reported outcome after ACL reconstruction based on data from the Scandinavian knee ligament registers.

\section{METHODS}

This systematic review was conducted according to the Preferred Reporting Items for Systematic Reviews and Meta-Analyses (PRISMA) guidelines. ${ }^{3}$

\section{Eligibility criteria}

All publications written in English from the Danish, Norwegian or Swedish knee ligament registers with a specific research question regarding ACL reconstruction were eligible for inclusion if the study presented data from the KOOS and/or European Quality of Life-5 dimensions (EQ-5D) and/or the Tegner Activity Scale. We excluded conference abstracts, review articles and clinical commentary articles. There were no prespecified patient demographics or type of ACL surgery intervention for the study populations.

\section{Information sources/literature search}

The literature search was performed by an expert in electronic search methods at the Sahlgrenska University Hospital library on 9 May 2018. An updated literature search was performed on 20 April 2018. We searched the MEDLINE/PubMed, EMBASE, The Cochrane Library and AMED electronic databases. Search terms were mapped to relevant $\mathrm{MeSH}$ terms or subject headings where possible. Search terms were entered into the database under three concepts: concept 1: 'Register', 'registry', 'registers' and 'registries'; concept 2: 'Sweden', 'Swedish', 'Denmark', 'Danish', 'Norway', 'Norwegian', 'Scandinavia', 'Scandinavian' and 'Nordic countries'; concept 3: 'Anterior 
cruciate ligament', 'Anterior cruciate ligament injuries', 'Anterior cruciate ligament reconstruction', 'Posterior cruciate ligament' and 'Posterior cruciate ligament reconstruction'. Keywords in each concept were grouped with the 'OR' operator. The results from each concept were then combined with the 'AND' operator to produce the search strategy and the final yield (see online supplementary table 1). In addition, the register holder of each Scandinavian knee ligament register was contacted via email and asked to provide a list of publications from the register. Two authors (EHS and ES) independently screened all abstracts and full texts, where needed, to identify eligible articles.

\section{Data collection process}

Data were extracted independently by two reviewers (EHS and ES). Disagreements were resolved by discussion, or by consulting with the senior author (KS). An electronic piloted form was created in Microsoft Excel for Windows (V.14.0.7, Microsoft Corp, Redmond, Washington, USA) for data collection.

\section{Outcome}

The KOOS is self-administered questionnaire validated for patients with knee injuries and knee osteoarthritis. ${ }^{2}$ It comprises 42 items across 5 subscales: pain (9 items), other symptoms (7 items), activities in daily living (ADL) (17 items), function in sport and recreation (5 items) and knee related quality of life (QoL) (4 items). Each subscale is scored on a 0 (worst) to 100 (best) scale.

The EQ-5D is a generic measurement of health status, although it has not been validated for patients who have sustained an ACL injury. The EQ-5D comprises 5 domains-mobility, self-care, usual activities, pain/discomfort and anxiety/depression-and a visual analogue scale (VAS) for overall health. ${ }^{4}$ An index value is calculated from the 5 domains, giving a score that ranges from 0 (worst) to 1 (best). The EQ-5D VAS is a vertical scale ranging from 0 (worst) to 100 (best). Both the EQ-5D index and EQ-5D VAS are reported in the Scandinavian knee ligament registers.

The Tegner Activity Scale range from 0 to 10, with higher scores corresponding to participation in knee strenuous activity. ${ }^{5}$ A patient participating in competitive sports at an elite level is considered to have a Tegner Activity Scale of 10. The Tegner Activity Scale also covers work related activities from level 0 to 5 , suggesting that all patients with a Tegner Activity Scale of 6 or higher are involved in sports.

\section{Risk of bias assessment}

There is no standardised method for assessing internal validity (bias) in register studies. We used the Downs and Black checklist for randomised and non-randomised studies. ${ }^{6}$ Two reviewers independently assessed each study. The original checklist comprises 27 items (each scored on a $0-2$ scale), and the maximum score is 30 . We excluded items $14,15,23$ and 24 because they are related to randomisation. We also excluded item 27 (power analysis), and item 21 (selection bias, as studies designed to analyse two or more registers would score zero and receive a lower score than a study investigating one register). The modified checklist comprised a maximum score of 22 points.

\section{Data synthesis}

Data synthesis was limited to a qualitative approach because of (1) overlapping participants in multiple studies meaning that data from some participants would have been included more than once in a quantitative analysis and (2) different methods (eg, follow-up lengths and statistical methods) used to address similar research questions and outcomes. A summary of the results from the original publications were synthesised under the following sections: patient related factors, surgery related factors and injury related factors. Furthermore, the results are summarised in the text under subheadings according to specific topics presented by the original studies (eg, 'hamstring tendon autograft versus patella tendon autograft'). In cases where the studies overlapped, the results from the study with the largest cohort were considered of highest evidence in the data synthesis, and secondarily, the study with the highest Downs and Black score was considered. No study was excluded due to overlapping cohorts. A summary of the extracted data is shown in the online supplementary table 2 .

\section{RESULTS}

The literature searches resulted in 157 individual studies for which the first round of screening was performed. One additional study was identified via the publication lists provided by the register holders. After applying the selection criteria, 35 studies were included in this systematic review (figure 1).

The quality score from the modified Downs and Black checklist ranged from 9 to 20 , with a median score of 16 points out of a possible 22 points (table 1). For some studies, the study design (cross sectional descriptive studies) made it difficult to apply many of the items and the reporting quality should be interpreted with care. ${ }^{7-9}$ Item 8 (adverse events reported) and item 19 (compliance reliable) were not fulfilled by any study. Twelve studies ensured representativeness of the recruited subjects (item 12), and 17 studies accounted for losses to follow-up (item 26).

\section{Relationship between patient related factors and patient reported outcomes \\ Patient sex}

Three studies from the Swedish National Knee Ligament Register (SNKLR) investigated differences in patient reported outcomes related to patient sex. ${ }^{71011}$ These studies had a quality score of 12-18 out of 22. At all follow-ups, women reported between 2 and 5 points lower than men in the KOOS sports and recreation $(\mathrm{P}<0.001) .{ }^{710}$ Women reported significantly less improvement in the KOOS sport and recreation from 1 to 2 years postoperatively compared with men (mean difference 3.2 [95\% CI 0.3 to 6.1]). ${ }^{10}$ Male sex has also been reported with favourable odds for functional recovery in KOOS (defined as KOOS pain $\geq 90$, symptoms $\geq 84, A D L \geq 91$, sport and recreation $\geq 80$ and QoL $\geq 81)$ compared with women (OR=1.37 [95\% CI 1.16 to 1.61], $\mathrm{P}<0.001) 2$ years after ACL reconstruction. ${ }^{11}$

\section{Age}

Three studies specifically studied the effect of patient age on patient reported outcome after ACL reconstruction. These studies had a quality score of 14-18 out of 22. Younger patients reported superior KOOS and Tegner Activity Scale ${ }^{10} 1213$ compared with older patients (>30 years) at all follow-ups. ${ }^{1012} 13$ However, the effect of age on patient reported outcome may be confounded by sex. ${ }^{10}$

\section{Smoking}

The effect of smoking on patient reported outcome was reported in two studies from the SNKLR. ${ }^{74}$ These studies had a quality score of 12 and 15 out of 22. Smokers reported inferior KOOS and EQ-5D outcomes at all follow-ups (preoperative, and 1, 2 and 5 years) compared with non-smokers, with the exception of the preoperative EQ-5D VAS. ${ }^{714}$ 


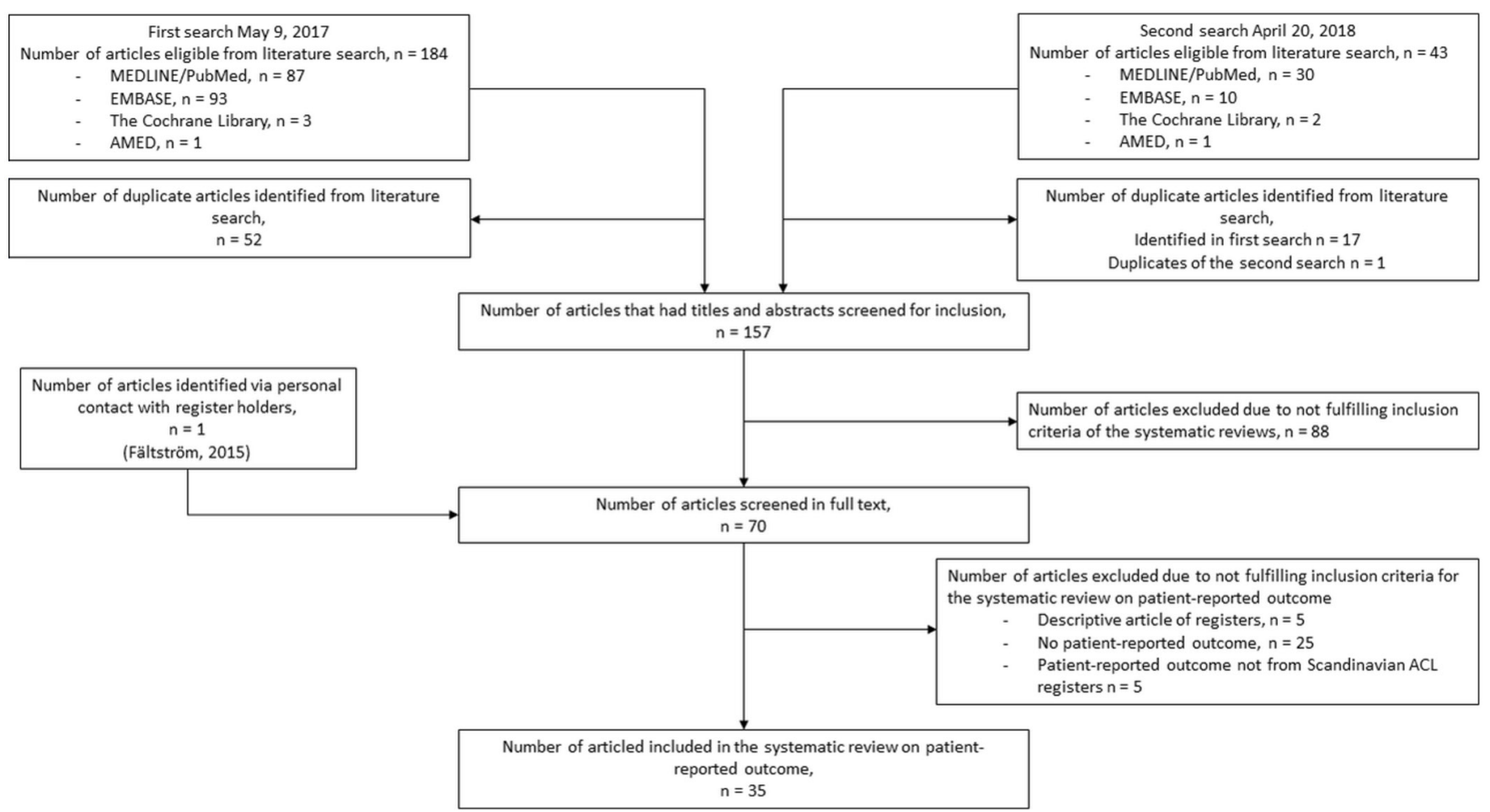

Figure 1 Article selection. ACL, anterior cruciate ligament.

Preoperative and postoperative rehabilitation

One study, with a quality score of 17 out of 22 , compared 84 patients who received preoperative and postoperative rehabilitation at a specialist clinic with 2690 matched patients from the Norwegian Knee Ligament Registry (NKLR) as a reflection of standard care. ${ }^{15}$ The active rehabilitation consisted of a specific training programme with recommendations for training dosage and exercises, which included heavy resistance strength training, plyometrics and neuromuscular exercises. The intervention cohort showed superior preoperative and postoperative KOOS for all subscales. Preoperatively, there were clinically relevant differences in all subscales, except in symptoms, and the largest difference was found in sport and recreation, with 24.6 points (95\% CI 19.0 to 30.2$)$. At the 2-year follow-up, clinically relevant differences were reported in KOOS symptoms, sport and recreation and QoL. The largest difference in the 2-year KOOS was reported in the subscales of sport and recreation (17.7 points [95\% CI 12.1 to 23.2$])^{15}$

\section{Interpretation of acceptable symptoms}

One study, with a quality score of 16 out of 22 , aimed to determine cutoffs and report the proportion of patients who had acceptable symptoms and treatment failure in the KOOS. With a total response rate of $62 \%$ at 6-24 months after ACL reconstruction, patients who reported acceptable symptoms scored between 76 and 91 points in the different KOOS subscales, while patients who reported treatment failure scored between 31 and 58 points. ${ }^{16}$ The proportion of patients who reported acceptable symptoms 6-24 months after ACL reconstruction was 55-66\% while $7-12 \%$ reported treatment failure.

\section{Minimal important change in the KOOS}

One study, with a quality score of 14 out of 22 , determined the minimal important change of the KOOS subscales for patients after ACL reconstruction up to 2 years. ${ }^{17}$ The minimal important change for KOOS pain was 2.5 points, KOOS symptoms was -1.2 points, KOOS ADL was 2.4 points, KOOS sport and recreation was 12.1 points and KOOS QoL was 18.3 points. The study also reported that $4-10 \%$ of patients may expect subscale specific worsening during the first 2 years after ACL reconstruction. ${ }^{17}$

\section{Non-response}

Two studies have compared responders and non-responders (ie, patients not responding to the patient reported outcomes administered by the registers) in the Scandinavian knee ligament registers. ${ }^{9}{ }^{18}$ These studies had a quality score of 14 and 19 out of 22. In both studies, non-responding patients were contacted and asked to reply to an additional assessment of patient reported outcomes (including the KOOS, EQ-5D and the Tegner Activity Scale), which was compared with responding patients. ${ }^{9}{ }^{18}$ No to minor differences were reported between patients responding and not responding to patient reported outcome preoperatively to 2 years after ACL reconstruction. ${ }^{18}$ However, younger patients and women had higher response rates compared with older patients and men. ${ }^{18}$

\section{Relationship between surgery related factors and patient reported outcomes}

Outcomes after primary and revision $\mathrm{ACL}$ reconstruction

Four studies have reported on outcomes after primary and revision ACL reconstruction, and how other surgical factors effect patient reported outcome after ACL reconstruction. ${ }^{8} 111920$ These studies had a quality score of 9 and 16 out of 22. Patients who underwent ACL reconstruction reported superior KOOS at 1, 2 and 5 years after reconstruction, compared with their preoperative KOOS. The 1- and 2-year KOOS, across all 


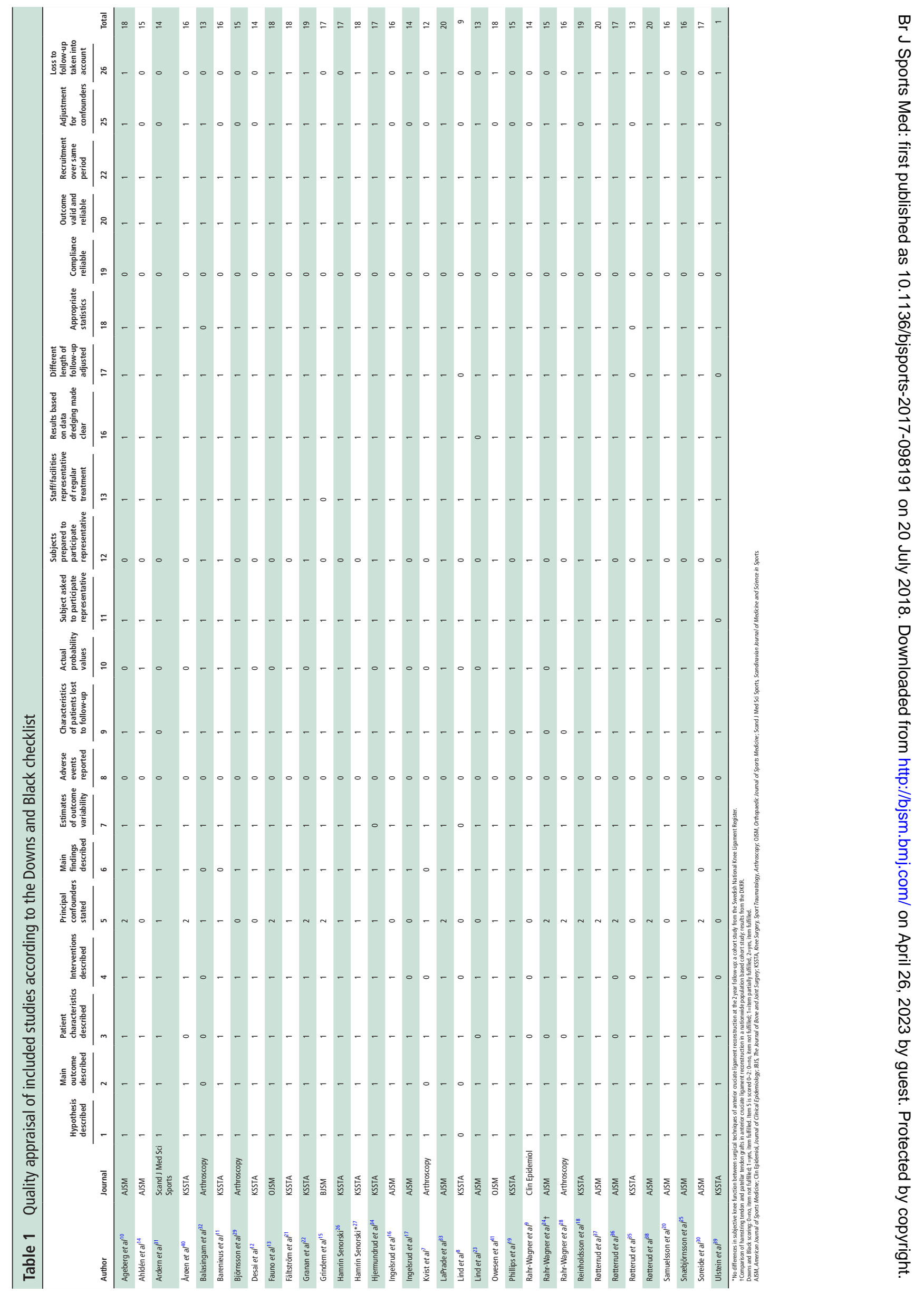


subscales, was equivalent after ACL reconstruction, independent of the potential confounders age, sex and concomitant injuries. ${ }^{20}$ Patients who waited $<3$ months from ACL injury to reconstruction reported superior KOOS in all subscales except symptoms, 2 years after reconstruction, compared with patients waiting $>3$ months. ${ }^{19}$ Patients who had notchplasty were less likely (OR $=0.68[95 \% \mathrm{CI} 0.56$ to $0.83, \mathrm{P}<0.01)$ to report functional recovery (defined as KOOS pain $\geq 90$, symptoms $\geq 84$, $\mathrm{ADL} \geq 91$, sport and recreation $\geq 80$ and $\mathrm{QoL} \geq 81$ ) 2 years after ACL reconstruction. ${ }^{11}$

Two studies have investigated if the patient reported outcome related to the primary ACL reconstruction could predict undergoing revision ACL reconstruction. ${ }^{21}{ }^{22}$ These studies had a quality score of 18 and 19 out of 22. A 2-year KOOS QoL $<44$ (proxy of treatment failure) increased the risk of undergoing revision ACL reconstruction $(\mathrm{RR}=3.7$ [95\% CI 2.2 to 6.0]), compared with patients with a KOOS QoL of $>44$ points. In addition, every 10 point reduction in KOOS QoL 2 years after the primary ACL increased the risk of revision ACL reconstruction by $33.6 \% .{ }^{22}$ Patients who subsequently underwent revision ACL reconstruction also reported inferior 2-year KOOS in the subscales of sport and recreation (19.5 points [95\% CI 13.4 to 25.7]) and QoL (15.8 points [95\% CI 10.4 to 21.3) compared with patients who did not. ${ }^{22}$ The risk of revision ACL reconstruction was also reduced by every 1 point increase in preoperative KOOS symptoms $(\mathrm{HR}=0.993[95 \%$ CI 0.989 to $0.998, \mathrm{P}=0.007)$, the $\mathrm{EQ}-5 \mathrm{D}$ index $(\mathrm{HR}=0.568$ [95\% CI 0.392 to 0.823$], \mathrm{P}=0.003$ ) and the EQ5-D VAS ( $\mathrm{HR}=0.994$ [95\% CI 0.991 to 0.998$], \mathrm{P}=0.005) .{ }^{21}$

Three studies have compared patient reported outcome after primary and revision ACL reconstruction. ${ }^{71423}$ These studies had a quality score of $12-15$ out of 22. Patients undergoing revision ACL reconstruction reported significantly inferior KOOS and EQ-5D on all follow-up occasions compared with those undergoing primary ACL reconstruction (with differences between 5 and 19 points in the KOOS). ${ }^{74}{ }^{143}$ A higher proportion of patients undergoing revision ACL reconstruction reported a KOOS QoL score $<40$ (as a proxy of treatment failure), $38 \%$ versus $20 \%$ of primary ACL reconstructions. ${ }^{23}$ However, patients who had revision ACL reconstruction reported improvements similar to primary reconstructions in all subscales of the KOOS, 1 and 2 years after surgery. ${ }^{23}$

\section{Contralateral and bilateral $\mathrm{ACL}$ reconstruction}

One study with a quality score of 18 out of 22 investigated preoperative KOOS as predictors for contralateral ACL reconstruction. ${ }^{21}$ There was a $0.6-1 \%$ increased risk of undergoing contralateral ACL reconstruction for every 1 point increase in all KOOS subscales preoperatively, with the exception of KOOS symptoms. ${ }^{21}$

One study with a quality score of 12 out of 22 compared the preoperative and 2-year KOOS between patients with bilateral ACL reconstruction and patients undergoing primary unilateral ACL reconstruction. ${ }^{7}$ No differences were reported preoperatively, however, at the 2-year follow-up, patients with bilateral reconstruction $(n=150)$ scored an average of 7 points lower across the KOOS subscales compared with patients with a unilateral ACL reconstruction $(\mathrm{n}=7652)(\mathrm{P}=0.008)$.

Hamstring tendon autograft versus patella tendon autograft Four studies compared patient reported outcomes between patients undergoing ACL reconstruction with either a hamstring tendon or a patella tendon autograft. ${ }^{7112425}$ These studies had a quality score of $12-16$ out of 22 . Patients who received a hamstring tendon autograft reported superior KOOS in the sport and recreation subscale (up to 7 points higher) at each follow-up compared with patients who received a patella tendon autograft. ${ }^{724}$ Patients who recieved a hamstring tendon autograft also had a higher Tegner Activity Scale compared with patients who recieved patella tendon autgograft (mean 4.9 [95\% CI 4.9 to 5.0] versus mean 4.7 [95\% CI 4.6 to 4.9 ]) 1 year postoperatively. ${ }^{24}$ Patients who received hamstring tendon autografts had increased odds of achieving functional recovery (defined as KOOS pain $\geq 90$, symptoms $\geq 84$, ADL $\geq 91$, sport and recreation $\geq 80$ and $\mathrm{QoL} \geq 81$ ) and were less likely to report treatment failure (defined as a KOOS QoL $<44$ points) compared with patients who received patella tendon autografts. ${ }^{11}$ Hamstring tendon graft diameter, stratified by $0.5 \mathrm{~mm}$ increments, did not affect KOOS or EQ-5D, pre- or postopertatively. ${ }^{25}$

\section{Femoral drilling and surgical technique}

Four studies investigated the effect of femoral tunnel drilling technique on patient reported outcome after ACL reconstruction. ${ }^{19}{ }^{26-28}$ These studies had a quality score of $15-18$ out of 22 . There was no difference in patient reported outcome (including the KOOS or Tegner Activity Scale) from preoperative to 2 years after primary ACL reconstruction with either the anteromedial portal or transportal compared with the transtibial femoral drilling technique. ${ }^{19} 2728$ Femoral tunnel drilling technique used in single-bundle ACL reconstruction did not predict which patients would report among the top or bottom $\mathrm{KOOS}_{4}$ quintiles 2 years after ACL reconstruction. ${ }^{26}$

\section{Double-bundle versus single-bundle $A C L$ reconstruction}

Three studies from the SNKLR compared patient reported outcome between double-bundle and single-bundle ACL reconstruction. ${ }^{71429}$ These studies had a quality score of $12-15$ out of 22. Patients who had a double-bundle ACL reconstruction reported lower preoperative KOOS compared with patients undergoing a single-bundle ACL reconstruction. ${ }^{7}{ }^{14} 29$ There were no differences between the groups at the 1 and 2 year follow-ups in KOOS or EQ-5D, in the improvement from the preoperative score to 1 year, or between 1 and 2 years postoperatively. ${ }^{29}$ However, patients who underwent double-bundle ACL reconstruction reported superior KOOS QoL at the 5 year follow-up. ${ }^{\top}$

\section{Non-steroidal anti-inflammatory drugs}

One study, with a quality score of 17 out of 22 , assessed the relationship between administration of non-steroidal anti-inflammatory drugs (NSAIDs) in ACL reconstruction and KOOS QoL at the 2-year follow-up. ${ }^{30}$ Patients administered NSAIDs reported superior KOOS across all subscales, and an overall reduced odds ratio (OR=0.8 [95\% CI 0.6 to 0.9$]$ ) of reporting a KOOS QoL subscale $<44$ at the 2-year follow-up compared with patients who were not administered NSAIDs. Further reduced odds of KOOS QoL $<44$ were found in the subgroup of patients aged $>29$ years $(\mathrm{OR}=0.7$ [95\% CI 0.5 to 0.9$])$ and men $(\mathrm{OR}=0.7$ [95\% CI 0.5 to 0.9$])$ who were administrated NSAIDs. ${ }^{30}$

\section{Non-reconstructive treatment}

One study, with a quality score of 14 out of 22 , compared KOOS after 1, 2 and 5 years of follow-up between patients who chose non-reconstructive treatment and patients undergoing ACL reconstruction (number of patients cross sectionally compared 
in the ACL reconstruction group and in the non-reconstructively treated group; 1 year KOOS $n=350,2$ year $n=358,5$ year $\mathrm{n}=114)$. ${ }^{31}$ Patients undergoing ACL reconstruction reported superior KOOS across all subscales and follow-ups.

\section{Relationship between injury related factors and patient reported outcome \\ Presence of concomitant injuries}

Three studies assessed the relationship between presence of concomitant injuries at ACL reconstruction and the KOOS. ${ }^{71432}$ These studies had a quality score of $12-15$ out of 22. Patients with concomitant intra-articular injuries (meniscus and articular cartilage injuries) reported inferior results in all KOOS subscales preoperatively and at the 1- and 2-year follow-ups compared with patients without concomitant injuries. ${ }^{714}$ Results at the 5-year follow-up were inconclusive although differences between patients with and without concomitant injuries appeared to decrease at this time point. ${ }^{74}{ }^{142}$ Differences between patients with concomitant injuries and isolated ACL reconstruction increased, superior for the latter, between the 5and 10-year follow-ups after reconstruction. However, both of these groups of patients reported slightly improved KOOS during these follow-ups. ${ }^{32}$

\section{Meniscal injuries}

Five studies assessed the relationship between concomitant meniscal injuries at ACL reconstruction and the KOOS. ${ }^{1114192633}$ These studies had a quality score of $15-20$ out and 22. Patients with concomitant meniscal injuries reported inferior KOOS preoperatively and at the 1-year follow-up for all subscales except for pain at 1 year, compared with those without meniscal injuries. At the 5-year follow-up, a significant difference was found in the subscale sport and recreation. ${ }^{14}$ The absence of meniscal injury increased the odds of reporting within the 80th percentile of $\mathrm{KOOS}_{4}, 2$ years after ACL reconstruction. ${ }^{26}$ There are inconclusive results on the effect of resection or repair of concomitant medial and lateral meniscus injuries compared with patients with isolated ACL reconstructions on 2-year KOOS..$^{193}$

Previous surgery to either the medial or lateral meniscus reduced the odds $(\mathrm{OR}=0.68$ [95\% CI 0.51 to 0.89], $\mathrm{P}=0.01$, and $\mathrm{OR}=0.53$ [ $95 \% \mathrm{CI} 0.36$ to 0.79 ], $\mathrm{P}<0.001$, respectively) of functional recovery (defined as KOOS pain $\geq 90$, symptoms $\geq 84, \mathrm{ADL} \geq 91$, sport and recreation $\geq 80$ and $\mathrm{QoL} \geq 81$ ) and increased the odds $(\mathrm{OR}=1.36$ [95\% CI 1.10 to 1.67], $\mathrm{P}=0.005$ and $\mathrm{OR}=1.42$ [95\% CI 1.08 to 1.86$], \mathrm{P}=0.01$, respectively) of treatment failure (defined as a KOOS QoL $<44$ points) 2 years after ACL reconstruction. ${ }^{11}$ There was increased odds of treatment failure (OR=1.28 [95\% CI 1.06 to 1.55$], \mathrm{P}=0.01$ ) with medial meniscus resection. ${ }^{11}$

\section{Articular cartilage injuries}

Eight studies investigated the effect of a concomitant articular cartilage injury on patient reported outcome after ACL reconstruction. ${ }^{19} 26$ 34-39 These studies had a quality score of 13-20 out of 22. The absence of articular cartilage injury increased the odds of reporting in the 80th percentile of $\mathrm{KOOS}_{4}, 2$ years after ACL reconstruction. ${ }^{26}$ Patients with full thickness cartilage lesions (ICRS grades 3-4) reported greater impairment on all KOOS subscales preoperatively and at the 1- and 2-year follow-ups compared with patients with partial-thickness lesions and patients without lesions. ${ }^{19}{ }^{34-37}$ There was no relationship between partial thickness cartilage lesions and poorer patient reported outcome at the 1- and 2-year follow-ups. ${ }^{35}$
One study reported that treatment with microfracture was associated with a negative effect on 2-year KOOS subscales of sport and recreation $(-8.6[95 \% \mathrm{CI}-16.4$ to -0.7$])$ and QoL $(-7.2$ [95\% CI -13.6 to -0.8$]$ ) compared with patient not receiving treatment for full thickness cartilage lesions. Debridement of full thickness cartilage lesions had no effect on patient reported outcomes compared with not recieving treatment. ${ }^{38}$

\section{Posterior cruciate ligament injury versus $\mathrm{ACL}$ injury}

Two studies have reported on the outcome after posterior cruciate ligament reconstruction (PCL) compared with ACL reconstruction. ${ }^{40} 41$ These studies had a quality score of 16 and 18 out of 22. Patients with an isolated PCL injury reported inferior preoperative and postoperative scores in all KOOS subscales compared with patients with an isolated ACL injury. Patients undergoing PCL reconstruction had similar improvements in KOOS as patients undergoing ACL reconstruction from preoperatively to 2 years after reconstruction. ${ }^{41}$

\section{DISCUSSION}

Modifiable factors that could affect patient reported outcome after ACL reconstruction include choosing hamstring tendon autograft over patella tendon autograft, not smoking, avoiding microfracture of full thickness concomitant cartilage injuries and rehabilitation treatment at a specialist clinic. The non-modifiable factors younger age at index ACL reconstruction and male sex were positively associated with patient reported outcomes while the presence of a full thickness cartilage injury and meniscal injuries were negatively associated with patient reported outcome.

The majority of findings relating to the KOOS were not clinically relevant, which suggest either that there are few meaningful differences between different patients and treatments, or that the outcome cannot discriminate between different patients and treatments. To minimise error and increase the relevance of the results obtained by using patient reported outcome, the outcome measurements should consist of condition specific items. ${ }^{42}$ There are concerns regarding the KOOS and its potential inadequate measurement properties in the three original WOMAC subscales (pain, symptoms and ADL) when used for patients after ACL reconstruction. ${ }^{42}{ }^{43}$ The risk of potential washout of treatment effects, type 1 errors and inadequate measurement properties when using an outcome which may not be optimally suited for the target population have been addressed by previous studies. ${ }^{20} 4244$ Because of this, clinicians are advised to read complementary literature to understand differences between patients and treatments, where population specific outcomes have been used-for example, literature where patients with and without concomitant meniscal injury are assessed with a meniscus relevant patient reported outcome.

\section{Patient related factors and patient reported outcome}

Younger patients and being male were the main patient related factors that positively affected patient reported outcome in the Scandinavian knee ligament registers, which is consistent with other scientific literature. ${ }^{71012-14}$ Younger patients may report superior KOOS pre- and postoperatively, but during the time between follow-ups, older patients ( $>40$ years) reported superior improvement in KOOS. ${ }^{12}$ Older patients may perceive a larger benefit from ACL reconstruction than younger patients, ${ }^{12}$ although this result can be confounded by the level of physical activity and motivation to return to sport.

It appears promising to combine data from the Scandinavian knee ligament register with other types of registers or databases 
as there has been an increase in studies using such methodology. ${ }^{15}{ }^{45-48}$ With such methodology, studies can include data on rehabilitation outcome, return to sport and the experience of surgeons and physiotherapists in order to address treatment related topics. For instance, no data on rehabilitation or compliance with treatment are kept in the Scandinavian knee ligament registers. Nevertheless, preoperative and postoperative subjective knee function were improved in patients who received specialised and controlled preoperative and postoperative rehabilitation (the Delaware-Oslo cohort) compared with standard care represented by the NKLR. ${ }^{15}$ In view of another register study, ${ }^{21}$ which reported that a lower preoperative KOOS symptoms was a predictor of revision ACL reconstruction, clinicians should advise patients to continue rehabilitation until an acceptable KOOS score occurs. In addition, patients who achieve sufficient knee function preoperatively reported superior outcomes after ACL reconstruction. ${ }^{49-51}$

\section{Treatment related factors and patient reported outcome}

Patients who underwent ACL reconstruction with hamstring tendon autografts reported superior results in the KOOS compared with patients with patella tendon autografts. ${ }^{7}$ This difference may be explained by the higher proportion of hamstring tendon autografts used in the registers ${ }^{52}$ and the more common donor site morbidity associated with the patella tendon autografts. ${ }^{53}$ In addition, Gifstad et al ${ }^{52}$ reported increased revision rates for hamstring tendon autografts compared with patella tendon autografts in younger patients participating in pivoting sports. These differences in patient reported knee funcation and revision rates have, however, not been confirmed by published randomised controlled trials comparing the two autograft choices in the short or long term follow-ups. ${ }^{545}$

To further understand and improve the care of patients with ACL injuries, patients who are treated without surgery should be included in national registers. Currently there are limited data. Patients treated non-reconstructively had inferior results on all KOOS subscales at all follow-ups. However, the only clinically meaningful difference was in the sport and recreation subscale at 1 year. ${ }^{56}$ In randomised trials using an evidence based rehabilitation protocol, patients treated non-reconstructively report similar results to patients treated with ACL reconstruction, ${ }^{5758}$ and there is likely selection bias in the registers. Therefore, the register based results should be interpreted with caution. There is a need for future research on the prognosis and indications for non-reconstructive treatment after ACL injury, where both randomised trials and large register studies are important to address this topic.

\section{Injury related factors and patient reported outcome}

Concomitant meniscal and articular cartilage injuries at ACL reconstruction are common in the Scandinavian knee ligament registers, most frequent in men and older patients. ${ }^{12}{ }^{1436}$ Patients who wait more than 1 year from ACL injury to surgery had a greater number of concomitant injuries. ${ }^{11}{ }^{59-61}$ Patients with concomitant injuries to menisci or articular cartilage reported inferior preoperative and early postoperative knee function compared with patients with an isolated ACL injury. ${ }^{14}$ However, the differences between patients with and without concomitant injuries do not reach clinical relevance at 5- and 10-year follow-up. ${ }^{32}$ Based on the registers, it is not known whether this can be explained by an adjustment in lifestyle and physical activity.
Surgical treatment of meniscal injury was associated with good short term outcomes. ${ }^{37}$ However, surgery to the lateral meniscus might have superior patient reported outcomes compared with surgery to the medial meniscus but the differences are small, which make the findings difficult to interpret. ${ }^{33}$ The differences between the presence of mensical injury or not, and meniscal treatment appear to decrease over time. This is important because it questions the discriminating ability of the KOOS as an outcome as patients with meniscal pathology have an increased risk of long term knee impairment and osteoarthritis, especially after meniscal resection, but this is not reflected by the outcome used in the registers. ${ }^{62-64}$

Concomitant cartilage lesions were also associated with inferior patient reported outcome..$^{35-3765}$ One common surgical treatment option for articular cartilage lesions is microfracture. Notable for clinicians, this procedure has been associated with poorer patient reported outcomes compared with other treatment options and no treatment. ${ }^{38}$

\section{Limitations}

We included all studies based on data from the Scandinavian knee ligament registers reporting on patient reported outcome. The results may be limited by only including level 2 and 3 studies, as randomised controlled trials are not possible to conduct based on registers. The large cohorts of the register studies can increase the risk of multiple significance. This means that the size and effect of the difference is important to consider, as a small difference may not be clinically relevant. Data were extracted from the original studies according to how the results were presented in each study and the absolute results were interpreted with results from studies which had a similar research question. This minimised loss of data but introduced potential limitations of bias in the interpretation of the results of the original studies.

As there is no risk of bias assessment tool specifically designed for register studies, we used a modified version of the Downs and Black checklist. All but one study had a score of 12-20 out of a possible score of 22 which indicates that studies from the Scandinavian knee ligament registers have good reporting quality. However, there are limitations in the Downs and Black checklist's assessment of internal validity (bias). Therefore, it is possible that we have inadequately accounted for the risk of bias and overstated the strength of the evidence. We did not define a threshold for high or low quality. The non-randomised design precludes inferences about causality. There is a high risk of attrition bias in this systematic review as only a small number of studies consider losses to follow-up.

A strength of the Scandinavian knee ligament registers is the high rate of coverage at baseline (proportion of participating units in relation to all eligible units) and completeness (proportion of target population in the register). Nevertheless, one of the largest potential limitations in the registers is the inadequate compliance of patient reported outcome follow-up, with a $50-70 \%$ response rate. ${ }^{79} 1866-69$ These numbers may be additionally biased by a higher response rate among women, older patients and those of higher socioeconomic status. ${ }^{918}$ However, validation and non-response analyses from the registers have not reported any differences in terms of patient reported outcomes. ${ }^{918}$

The numerous studies from the Scandinavian knee ligament registers create a strong foundation for a high level of evidence, but the frequent publication also results in a potential bias of using the same cohort and patient data for analysis. In order to avoid the potential bias of using the same patient's data 


\section{What is already known on this topic?}

- The Scandinavian knee ligament registers enable identification of surgical techniques and factors that may lead to premature failure, as well as predictors for good and poor outcomes.

- Over $90 \%$ of the annually performed anterior cruciate ligament $(\mathrm{ACL})$ reconstructions are registered and the data are strengthened by prospective collection.

- The patient reported outcome within the Scandinavian knee ligament registers are valuable for detection of inferior results and early failures in patients undergoing $\mathrm{ACL}$ reconstruction.

multiple times, the synthesis of data in this systematic review was performed by interpreting the results of studies addressing similar research questions summarised under three sections.

\section{CONCLUSION}

Both primary and revision ACL reconstruction improve subjective knee function compared with preoperative status. Younger age, male sex, not smoking, receiving hamstring tendon autografts and having no concomitant injuries were associated with superior patient reported outcomes. There were no clinically relevant differences in patient reported knee function with regard to the ACL reconstruction techniques used.

Acknowledgements The authors thank biostatistician Therese Svanberg from the Sahlgrenska University Hospital library for help with the electronic search of this systematic review.

Contributors EHS, ES and KS contributed substantially to the acquisition of the data, analysis of the data and are responsible for drafting the work and revising it critically for important intellectual content. AB, FF and ORA made substantial contributions to the conception and design of the work, including interpretation of the data. LE and JK made large contributions to the revision and design of the manuscript. All authors have given their final approval of the manuscript to be published. In addition, all authors are in agreement to be accountable for all aspects of the work in ensuring that questions related to the accuracy or integrity of any part of the work are appropriately investigated and resolved.

Funding The authors have not declared a specific grant for this research from any funding agency in the public, commercial or not-for-profit sectors.

Competing interests None declared.

Patient consent Not required.

Provenance and peer review Not commissioned; externally peer reviewed.

\section{REFERENCES}

1 Prentice HA, Lind M, Mouton C, et al. Patient demographic and surgical characteristics in anterior cruciate ligament reconstruction: a description of registries from six countries. Br J Sports Med 2018;52:716-22.

\section{What are the new findings?}

- The information acquired from registers is of high generalisability and can facilitate clinicians' treatment decisions for the individualised management of $\mathrm{ACL}$ injuries.

- Clinicians should be aware of modifiable factors that may influence patient reported outcome, and should adjust treatment and rehabilitation with emphasis on targeting these factors.

- Clinicians should be aware that the non-modifiable factors identified highlight specific subgroups of patients who may require additional treatment.
2 Roos EM, Roos HP, Lohmander LS, et al. Knee Injury and Osteoarthritis Outcome Score (KOOS)-development of a self-administered outcome measure. J Orthop Sports Phys Ther 1998;28:88-96.

3 Stewart LA, Clarke M, Rovers M, et al. Preferred reporting items for systematic review and meta-analyses of individual participant data: the PRISMA-IPD Statement. JAMA 2015;313:1657-65.

4 EuroQol Group. EuroQol-a new facility for the measurement of health-related quality of life. Health Policy 1990;16:199-208.

5 Tegner Y, Lysholm J. Rating systems in the evaluation of knee ligament injuries. Clin Orthop Relat Res 1985;198:43-9.

6 Downs SH, Black N. The feasibility of creating a checklist for the assessment of the methodological quality both of randomised and non-randomised studies of health care interventions. J Epidemiol Community Health 1998;52:377-84.

7 Kvist J, Kartus J, Karlsson J, et al. Results from the Swedish National Anterior Cruciate Ligament Register. Arthroscopy 2014;30:803-10.

8 Lind M, Menhert F, Pedersen AB. The first results from the Danish ACL reconstruction registry: epidemiologic and 2 year follow-up results from 5,818 knee ligament reconstructions. Knee Surg Sports Traumatol Arthrosc 2009;17:117-24.

9 Rahr-Wagner L, Thillemann TM, Lind MC, et al. Validation of 14,500 operated knees registered in the Danish Knee Ligament Reconstruction Register: registration completeness and validity of key variables. Clin Epidemiol 2013;5:219-28.

10 Ageberg E, Forssblad M, Herbertsson P, et al. Sex differences in patient-reported outcomes after anterior cruciate ligament reconstruction: data from the Swedish Knee Ligament Register. Am J Sports Med 2010;38:1334-42.

11 Barenius B, Forssblad M, Engström B, et al. Functional recovery after anterior cruciate ligament reconstruction, a study of health-related quality of life based on the Swedish National Knee Ligament Register. Knee Surg Sports Traumatol Arthrosc 2013:21:914-27.

12 Desai N, Björnsson H, Samuelsson K, et al. Outcomes after ACL reconstruction with focus on older patients: results from The Swedish National Anterior Cruciate Ligament Register. Knee Surg Sports Traumatol Arthrosc 2014;22:379-86.

13 Faunø P, Rahr-Wagner L, Lind M. Risk for revision after anterior cruciate ligament reconstruction is higher among adolescents: results from the Danish registry of knee ligament reconstruction. Orthop J Sports Med 2014;2:2325967114552405.

14 Ahldén M, Samuelsson K, Sernert N, et al. The Swedish national anterior cruciate ligament register: a report on baseline variables and outcomes of surgery for almost 18,000 patients. Am J Sports Med 2012;40:2230-5.

15 Grindem H, Granan LP, Risberg MA, et al. How does a combined preoperative and postoperative rehabilitation programme influence the outcome of $A C L$ reconstruction 2 years after surgery? A comparison between patients in the Delaware-Oslo ACL Cohort and the Norwegian National Knee Ligament Registry. Br I Sports Med 2015;49:385-9.

16 Ingelsrud LH, Granan LP, Terwee CB, et al. Proportion of patients reporting acceptable symptoms or treatment failure and their associated KOOS values at 6 to 24 months after anterior cruciate ligament reconstruction: a study from the Norwegian Knee Ligament Registry. Am J Sports Med 2015;43:1902-7.

17 Ingelsrud $\mathrm{LH}$, Terwee $\mathrm{CB}$, Terluin $\mathrm{B}$, et al. Meaningful change scores in the knee injury and osteoarthritis outcome score in patients undergoing anterior cruciate ligament reconstruction. Am J Sports Med 2018;46:1120-8.

18 Reinholdsson J, Kraus-Schmitz J, Forssblad M, et al. A non-response analysis of 2-year data in the Swedish Knee Ligament Register. Knee Surg Sports Traumatol Arthrosc 2017:25:2481-7.

19 Phillips M, Rönnblad E, Lopez-Rengstig L, et al. Meniscus repair with simultaneous ACL reconstruction demonstrated similar clinical outcomes as isolated $A C L$ repair: a result not seen with meniscus resection. Knee Surg Sports Traumatol Arthrosc 2018.

20 Samuelsson K, Magnussen RA, Alentorn-Geli E, et al. Equivalent knee injury and osteoarthritis outcome scores 12 and 24 months after anterior cruciate ligament reconstruction: results from the Swedish National Knee Ligament Register. Am J Sports Med 2017;45:2085-91.

21 Fältström A, Hägglund M, Magnusson $\mathrm{H}$, et al. Predictors for additional anterior cruciate ligament reconstruction: data from the Swedish National ACL Register. Knee Surg Sports Traumatol Arthrosc 2016;24:885-94.

22 Granan LP, Baste V, Engebretsen L, et al. Associations between inadequate knee function detected by KOOS and prospective graft failure in an anterior cruciate ligament-reconstructed knee. Knee Surg Sports Traumatol Arthrosc 2015;23:1135-40.

23 Lind $\mathrm{M}$, Menhert F, Pedersen AB. Incidence and outcome after revision anterior cruciate ligament reconstruction: results from the Danish registry for knee ligament reconstructions. Am J Sports Med 2012;40:1551-7.

24 Rahr-Wagner L, Thillemann TM, Pedersen AB, et al. Comparison of hamstring tendon and patellar tendon grafts in anterior cruciate ligament reconstruction in a nationwide population-based cohort study: results from the Danish registry of knee ligament reconstruction. Am J Sports Med 2014;42:278-84.

25 Snaebjörnsson T, Hamrin Senorski E, Ayeni OR, et al. Graft diameter as a predictor for revision anterior cruciate ligament reconstruction and KOOS and EQ-5D values: a cohort study from the Swedish National Knee Ligament Register based on 2240 patients. Am J Sports Med 2017;45:2092-7.

26 Hamrin Senorski E, Alentorn-Geli E, Musahl V, et al. Increased odds of patientreported success at 2 years after anterior cruciate ligament reconstruction in patients 
without cartilage lesions: a cohort study from the Swedish National Knee Ligament Register. Knee Surg Sports Traumatol Arthrosc 2018;26:1086-95.

27 Hamrin Senorski E, Sundemo D, Murawski CD, et al. No differences in subjective knee function between surgical techniques of anterior cruciate ligament reconstruction at 2-year follow-up: a cohort study from the Swedish National Knee Ligament Register. Knee Surg Sports Traumatol Arthrosc 2017;25:3945-54.

28 Rahr-Wagner L, Thillemann TM, Pedersen AB, et al. Increased risk of revision after anteromedial compared with transtibial drilling of the femoral tunnel during primary anterior cruciate ligament reconstruction: results from the Danish Knee Ligament Reconstruction Register. Arthroscopy 2013;29:98-105.

29 Björnsson $\mathrm{H}$, Andernord $\mathrm{D}$, Desai $\mathrm{N}$, et al. No difference in revision rates between single- and double-bundle anterior cruciate ligament reconstruction: a comparative study of 16,791 patients from the Swedish National Knee Ligament Register. Arthroscopy 2015;31:659-64.

30 Soreide E, Granan LP, Hjorthaug GA, et al. The effect of limited perioperative nonsteroidal anti-inflammatory drugs on patients undergoing anterior cruciate ligament reconstruction. Am J Sports Med 2016;44:3111-8.

31 Ardern CL, Sonesson S, Forssblad M, et al. Comparison of patient-reported outcomes among those who chose ACL reconstruction or non-surgical treatment. Scand I Med Sci Sports 2017;27:535-44

32 Balasingam S, Sernert N, Magnusson H, et al. Patients with concomitant intra-articular lesions at index surgery deteriorate in their knee injury and osteoarthritis outcome score in the long term more than patients with isolated anterior cruciate ligament rupture: a study from the Swedish National Anterior Cruciate Ligament Register. Arthroscopy 2018;34:1520-9.

33 LaPrade CM, Dornan GJ, Granan LP, et al. Outcomes after anterior cruciate ligament reconstruction using the Norwegian Knee Ligament Registry of 4691 patients: how does meniscal repair or resection affect short-term outcomes? Am I Sports Med 2015;43:1591-7.

34 Hjermundrud V, Bjune TK, Risberg MA, et al. Full-thickness cartilage lesion do not affect knee function in patients with ACL injury. Knee Surg Sports Traumatol Arthrosc 2010:18:298-303

35 Røtterud JH, Risberg MA, Engebretsen L, et al. Patients with focal full-thickness cartilage lesions benefit less from ACL reconstruction at 2-5 years follow-up. Knee Surg Sports Traumatol Arthrosc 2012;20:1533-9.

36 Røtterud JH, Sivertsen EA, Forssblad M, et al. Effect of gender and sports on the risk of full-thickness articular cartilage lesions in anterior cruciate ligament-injured knees: a nationwide cohort study from Sweden and Norway of 15783 patients. Am I Sports Med 2011;39:1387-94.

37 Røtterud JH, Sivertsen EA, Forssblad M, et al. Effect of meniscal and focal cartilage lesions on patient-reported outcome after anterior cruciate ligament reconstruction: a nationwide cohort study from Norway and Sweden of 8476 patients with 2-year follow-up. Am J Sports Med 2013;41:535-43.

38 Røtterud JH, Sivertsen EA, Forssblad M, et al. Effect on patient-reported outcomes of debridement or microfracture of concomitant full-thickness cartilage lesions in anterior cruciate ligament-reconstructed knees: a nationwide cohort study from Norway and Sweden of 357 Patients With 2-Year Follow-up. Am I Sports Med 2016:44:337-44.

39 Ulstein S, Bredland K, Årøen A, et al. No negative effect on patient-reported outcome of concomitant cartilage lesions 5-9 years after ACL reconstruction. Knee Surg Sports Traumatol Arthrosc 2017;25:1482-8.

40 Arøen A, Sivertsen EA, Owesen C, et al. An isolated rupture of the posterior cruciate ligament results in reduced preoperative knee function in comparison with an anterior cruciate ligament injury. Knee Surg Sports Traumatol Arthrosc 2013:21:1017-22.

41 Owesen C, Sivertsen EA, Engebretsen L, et al. Patients with isolated PCL injuries improve from surgery as much as patients with $\mathrm{ACL}$ injuries after 2 years. Orthop J Sports Med 2015;3:232596711559953.

42 Comins J, Brodersen J, Krogsgaard M, et al. Rasch analysis of the Knee injury and Osteoarthritis Outcome Score (KOOS): a statistical re-evaluation. Scand J Med SCi Sports 2008; 18:336-45.

43 Krogsgaard MR, Brodersen J, Comins J. A scientific approach to optimal treatment of cruciate ligament injuries. Acta Orthop 2011;82:389-90. discussion 90-2.

44 Spindler KP, Huston LJ, Chagin KM, et al. Ten-Year Outcomes and Risk Factors After Anterior Cruciate Ligament Reconstruction: A MOON Longitudinal Prospective Cohort Study. Am J Sports Med 2018;46:815-25.

45 Fältström A, Hägglund M, Kvist J. Factors associated with playing football after anterior cruciate ligament reconstruction in female football players. Scand J Med Sci Sports 2016;26:1343-52.
46 Hamrin Senorski E, Svantesson E, Beischer S, et al. Concomitant injuries may not reduce the likelihood of achieving symmetrical muscle function one year after anterior cruciate ligament reconstruction: a prospective observational study based on 263 patients. Knee Surg Sports Traumatol Arthrosc 2018;40.

47 Hamrin Senorski E, Svantesson E, Beischer S, et al. Low 1-year return-to-sport rate after anterior cruciate ligament reconstruction regardless of patient and surgical factors: a prospective cohort study of 272 patients. Am I Sports Med 2018;46:1551-8.

48 Rahr-Wagner L, Thillemann TM, Lind M, et al. Comorbidities in patients with anterior cruciate ligament reconstruction compared with matched controls without anterior cruciate ligament injury from danish registries. Arthroscopy 2015;31:1741-7.

49 Adams D, Logerstedt DS, Hunter-Giordano A, et al. Current concepts for anterior cruciate ligament reconstruction: a criterion-based rehabilitation progression. J Orthop Sports Phys Ther 2012;42:601-14.

50 Eitzen I, Holm I, Risberg MA. Preoperative quadriceps strength is a significant predictor of knee function two years after anterior cruciate ligament reconstruction. $\mathrm{Br}$ J Sports Med 2009;43:371-6.

51 Risberg MA, Grindem H, Øiestad BE. We need to implement current evidence in early rehabilitation programs to improve long-term outcome after anterior cruciate ligament injury. J Orthop Sports Phys Ther 2016;46:710-3.

52 Gifstad T, Foss OA, Engebretsen L, et al. Lower risk of revision with patellar tendon autografts compared with hamstring autografts: a registry study based on 45,998 primary ACL reconstructions in Scandinavia. Am J Sports Med 2014;42:2319-28.

53 Webster KE, Feller JA, Hartnett N, et al. Comparison of patellar tendon and hamstring tendon anterior cruciate ligament reconstruction: a 15-year follow-up of a randomized controlled trial. Am J Sports Med 2016;44:83-90.

54 Mohtadi NG, Chan DS, Dainty KN, et al. Patellar tendon versus hamstring tendon autograft for anterior cruciate ligament rupture in adults. Cochrane Database Syst Rev 2011;9:Cd005960.

55 Samuelsen BT, Webster KE, Johnson NR, et al. Hamstring autograft versus patellar tendon autograft for $\mathrm{ACL}$ reconstruction: is there a difference in graft failure rate? $\mathrm{A}$ meta-analysis of 47,613 Patients. Clin Orthop Relat Res 2017;475:2459-68.

56 Roos EM, Lohmander LS. The Knee injury and Osteoarthritis Outcome Score (KOOS): from joint injury to osteoarthritis. Health Qual Life Outcomes 2003;1:64.

57 Frobell RB, Roos EM, Roos HP, et al. A randomized trial of treatment for acute anterior cruciate ligament tears. N Engl J Med 2010;363:331-42.

58 Peccin MS, Almeida GJ, Amaro J, et al. Interventions for treating posterior cruciate ligament injuries of the knee in adults. Cochrane Database Syst Rev 2005;2:Cd002939.

59 Andernord D, Björnsson H, Petzold M, et al. Surgical predictors of early revision surgery after anterior cruciate ligament reconstruction: results from the Swedish National Knee Ligament Register on 13,102 Patients. Am J Sports Med 2014;42:1574-82.

60 Andernord D, Desai N, Björnsson $\mathrm{H}$, et al. Patient predictors of early revision surgery after anterior cruciate ligament reconstruction: a cohort study of 16,930 patients with 2-year follow-up. Am J Sports Med 2015;43:121-7.

61 Granan LP, Bahr R, Lie SA, et al. Timing of anterior cruciate ligament reconstructive surgery and risk of cartilage lesions and meniscal tears: a cohort study based on the Norwegian National Knee Ligament Registry. Am J Sports Med 2009;37:955-61.

62 Ajuied A, Wong F, Smith C, et al. Anterior cruciate ligament injury and radiologic progression of knee osteoarthritis: a systematic review and meta-analysis. Am I Sports Med 2014:42:2242-52.

63 Øiestad BE, Engebretsen L, Storheim K, et al. Knee osteoarthritis after anterior cruciate ligament injury: a systematic review. Am I Sports Med 2009;37:1434-43.

64 Risberg MA, Oiestad BE, Gunderson R, et al. Changes in knee osteoarthritis, symptoms, and function after anterior cruciate ligament reconstruction: a 20-year prospective follow-up study. Am J Sports Med 2016;44:1215-24.

65 Årøen A, Brøgger H, Røtterud JH, et al. Evaluation of focal cartilage lesions of the knee using MRI T2 mapping and delayed Gadolinium Enhanced MRI of Cartilage (dGEMRIC). BMC Musculoskelet Disord 2016;17:73.

66 Emilsson L, Lindahl B, Köster M, et al. Review of 103 Swedish healthcare quality registries. J Intern Med 2015;277:94-136.

67 Engebretsen L, Forssblad M, Lind M. Why registries analysing cruciate ligament surgery are important. Br J Sports Med 2015;49:636-8.

68 Granan LP, Bahr R, Steindal K, et al. Development of a national cruciate ligament surgery registry: the Norwegian National Knee Ligament Registry. Am J Sports Med 2008;36:308-15

69 Rahr-Wagner L, Lind M. The Danish Knee Ligament Reconstruction Registry. Clin Epidemiol 2016;8:531-5. 